\title{
In-Hospital Transfer Is a Risk Factor for Invasive Filamentous Fungal Infection among Hospitalized Patients with Hematological Malignancies: A Matched Case-Control Study
}

\author{
Angèle Gayet-Ageron; ${ }^{1,2, \star}$ Anne Iten; ${ }^{1, \star}$ Christian van Delden; ${ }^{3}$ Natacha Farquet; ${ }^{1}$ Stavroula Masouridi-Levrat; ${ }^{4}$ \\ Elodie Von Dach; ${ }^{1}$ Yves Chalandon; ${ }^{4}$ Didier Pittet ${ }^{1}$
}

ов јестіле. Immunocompromised patients now benefit from a longer life expectancy due to advanced medical techniques, but they are also weakened by aggressive treatment approaches and are at high risk for invasive fungal disease. We determined risk factors associated with an outbreak of invasive filamentous fungal infection (IFFI) among hospitalized hemato-oncological patients.

Methods. A retrospective, matched, case-control study was conducted between January 1, 2009, and April 31, 2011, including 29 cases ( 6 proven, 8 probable, and 15 possible) of IFFI and 102 matched control patients hospitalized during the same time period. Control patients were identified from the hospital electronic database. Conditional logistic regression was performed to identify independent risk factors for IFFI.

RESULTS. Overall mortality associated with IFFI was 20.7\% (8.0\%-39.7\%). Myelodysplastic syndrome was associated with a higher risk for IFFI compared to chronic hematological malignancies. After adjustment for major risk factors and confounders, $>5$ patient transfers outside the protected environment of the hematology ward increased the IFFI risk by 6.1 -fold. The risk increased by 6.7 -fold when transfers were performed during neutropenia.

CONCLUSION. This IFFI outbreak was characterized by a strong association with exposure to the unprotected environment outside the hematology ward during patient transfer. The independent associations of a high number of transfers with the presence of neutropenia suggest that affected patients were probably not sufficiently protected during transport in the corridors. Our study highlights that a heightened awareness of the need for preventive measures during the entire care process of at-risk patients should be promoted among healthcare workers.

Infect Control Hosp Epidemiol 2015;36(3):320-328

Invasive filamentous fungal infection (IFFI) is a devastating opportunistic infection responsible for a large number of complications and deaths among immunocompromised patients. ${ }^{1,2}$ The ubiquitous presence of filamentous fungi in the environment, such as Aspergillus species, has the potential to lead to IFFI in the severely immunocompromised, particularly patients with hematological malignancies, ${ }^{3}$ patients undergoing hematopoietic stem cell transplant (HSCT), or solid organ transplant recipients. ${ }^{4}$ The reported mortality rate of IFFI ranges from $50 \%$ in patients with leukemia or lymphoma to $87 \%$ in HSCT patients. ${ }^{1}$ IFFI is a constant preoccupation in immunocompromised patients regularly hospitalized and exposed to aggressive treatments. Apart from individual risk factors, environmental factors (eg, hot windy weather, high relative humidity, or the presence of construction work in and around medical care facilities) increase the concentration of airborne fungal spores and the risk for IFFI among susceptible patients. ${ }^{5-7}$ Routine surveillance and systematic investigation of suspected outbreaks are crucial to identifying failures in system care management and the proposal of targeted corrective measures.

Based on a low incidence of IFFI at our institution, ${ }^{8-10}$ we investigated a suspected outbreak following the occurrence of a cluster of cases during summer 2010 in 2 wards housing

Preliminary results from this study were presented at the $2^{\text {nd }}$ International Conference on Prevention and Infection Control; June 25-28, 2013, Geneva, Switzerland, Poster 202 (June 20, 2013).

Affiliations: 1. Infection Control Program and WHO Collaborating Centre on Patient Safety, University of Geneva Hospitals and Faculty of Medicine, Geneva, Switzerland; 2. Clinical Research Center \& Division of Clinical Epidemiology, Department of Health and Community Medicine, University of Geneva Hospitals and Faculty of Medicine, Geneva, Switzerland; 3. Division of Infectious Diseases, University of Geneva Hospitals and Faculty of Medicine, Geneva, Switzerland; 4. Division of Hematology,University of Geneva Hospitals and Faculty of Medicine, Geneva, Switzerland.

${ }^{*} \mathrm{AGA}$ and $\mathrm{AI}$ contributed equally to this manuscript.

Received August 8, 2014; accepted November 25, 2014.; electronically published January 9, 2015

(c) 2015 by The Society for Healthcare Epidemiology of America. All rights reserved. 0899-823X/2015/3603-0012. DOI: 10.1017/ice.2014.69 
patients admitted for hematological malignancies. The purpose of this retrospective, matched case-control study was to identify risk factors associated with IFFI.

\section{METHODS}

\section{Study Design, Setting, and Population}

A retrospective, matched case-control study nested in a cohort of at-risk inpatients was performed between January 1, 2009, and April 31, 2011, at the University of Geneva Hospitals, Switzerland, an 1,800-bed tertiary healthcare center. Patients undergoing allogeneic HSCT and those with hematological malignancies are housed in 2 specialized hospital wards located on the 5th (HSCT ward) and 7th floors (non-HSCT ward), respectively.

\section{Case Definition}

We defined "proven," "probable," or "possible" IFFI according to standardized definitions. ${ }^{11}$ All case definitions were assessed by 2 investigators (CVD, YC).

\section{Control Definition}

Control patients were randomly selected from a list of patients presenting predefined at-risk disease (ie, hematological malignancies or bone-marrow failure syndromes) coded according to the World Health Organization International Classification of Diseases, version 10, or allogeneic HSCT recipients. This list was obtained from the institutional electronic record database of patients hospitalized during the study period. The first 5 controls were randomly selected for the same period of hospitalization before IFFI diagnosis in the case ( \pm 2 months) to assess risk factors other than construction work. Exclusion criteria for control patients were the presence of IFFI before and during the outbreak.

\section{Variables and Data Sources}

Individual characteristics were extracted from patient records and included a history of cytomegalovirus infection, pulmonary disease, past severe neutropenia, active neutropenia during hospital stay, underlying diseases, and prescription of antifungal prophylaxis and/or immunosuppressive therapy. Chemotherapy protocols were classified into 4 categories (from highly cytotoxic to less cytotoxic), and the variable was dichotomized into highly cytotoxic versus moderately to not cytotoxic, or no chemotherapy. Underlying hematological diseases were classified in 3 groups: (1) acute disease (eg, acute myeloid leukemia, acute lymphoblastic leukemia or bone marrow failure syndrome); (2) chronic disease (eg, chronic lymphatic leukemia, chronic myeloid leukemia, non-Hodgkin lymphoma, Hodgkin lymphoma, multiple myeloma, or myeloproliferative neoplasms); and (3) myelodysplastic syndrome. All variables were predefined in a dedicated case report form completed by 4 investigators.
Between August 2008 and April 2011, several construction and renovation projects were initiated at our hospital, including the complete demolition and (ongoing) reconstruction of an 8-floor block adjoining the main hospital building. Although specific measures were introduced as recommended, ${ }^{12-14}$ we suspected that transport from the protected environment to other hospital areas might represent a risk factor for IFFI. To evaluate this hypothesis, we obtained the number of transfers as a proxy of construction work exposure from the electronic patient database (ie, date, time, and place of transfer).

We considered 3 study periods: (1) "before the epidemic" (January 1, 2007, through December 31, 2008); (2) "during the epidemic" (January 1, 2009, through April 31, 2011) corresponding to the end of massive construction works; and (3) "after the epidemic" (May 1, 2011, through May 31, 2013).

\section{Environmental Surveillance}

Air and surface sampling for bacterial and fungal counts in the protected wards have been monitored monthly at our institution since 1992. For the purpose of this study, we used data from the active surveillance system before and after the suspected outbreak, (ie, between January 1, 2007, and December 31, 2013). Methods used for air, floor, and other horizontal surface sampling are described elsewhere. ${ }^{9}$ Results are expressed as colony-forming units per cubic centimeter $\left(\mathrm{cfu} / \mathrm{m}^{3}\right)$ for air and colony-forming units per 25 square centimeters $\left(\mathrm{cfu} / 25 \mathrm{~cm}^{2}\right)$ for surfaces.

\section{Antifungal Prophylaxis and Empirical Treatment Strategies}

Standard care in both wards included antifungal prophylaxis with oral fluconazole $(200 \mathrm{mg} /$ day). Oral voriconazole was used for empirical antifungal therapy for fever persisting $>5$ days. Thoracic CT scans were performed when clinically indicated or for persisting fever, and serum galactomannan levels were monitored weekly.

\section{Cost Analysis}

Direct costs attributed to the outbreak included a switch from fluconazole to antifungal prophylaxis covering filamentous fungi. Costs related to additional preventive control measures targeted at patients, visitors, and healthcare workers (ie, caps, gowns, masks), environmental measures (ie, in-depth room cleaning), and equipment costs (ie, door closure systems, information brochures, and transport trolley). Direct costs did not include the time and salaries of the investigators of the outbreak.

\section{Statistical Analysis}

The total number of cases of IFFI was 29 . We estimated that $50 \%$ of control patients would have $>1$ transfer during his or 
her hospital stay and that the correlation coefficient for exposure between matched cases and controls would be $0.45 .{ }^{15}$ The research hypothesis was a 4.5 -fold increase in the odds of having IFFI in the presence of $\geq 5$ transfers. With 5 controls per case and an $\alpha$ error of $5 \%$, the power of the study to show an association was $80 \%$.

All variables associated with an increased likelihood of IFFI in univariate analysis at a significance level of $P<.25$ were included in a multivariate conditional logistic regression model using a forward stepwise procedure. For continuous variables, we verified whether log-linearity was respected; when it was not respected, variables were presented as categorical. In the final multivariate model, we included a limited number of risk factors to respect the number of events per variable,${ }^{16}$ but we forced into the model the number of transfers and length of hospital stay, which are correlated with the primary predictor. We performed the same analyses after the exclusion of "possible" cases. We present odds ratios (ORs) and their $95 \%$ confidence intervals (95\% CIs). The percentage of variance in outcome explained by the model was estimated by calculating a pseudo- $\mathrm{R}^{2}$ value.

For environmental data, we used a non-parametric KruskalWallis test to assess whether mean air total counts and Aspergillus spp. growth in air cultures were statistically significant depending on the 3 study periods and stratified by ward. We applied a Poisson regression to determine total growth or the number of filamentous fungi using an interaction term between study periods and the ward tested. A 2-sided $P$ value $<.05$ was considered statistically significant. The analyses were performed using Stata intercooled IC 13.0 (StataCorp, College Station, TX).

\section{RES ULTS}

\section{Case Descriptions}

A total of 29 cases were diagnosed between January 1, 2009, and April 31, 2011 (Figure 1). Among these, 6 cases were defined as proven IFFIs (20.7\%), 8 as probable IFFIs (27.6\%), and 15 as possible IFFIs (51.7\%). Most were diagnosed during summer 2010 when the alert was raised. Overall mortality was $20.7 \%$ (95\% CI, 8.0-39.7). A total of 6 deaths occurred among proven cases $(\mathrm{n}=4 ; 66.7 \%)$ and probable cases $(\mathrm{n}=2 ; 33.3 \%)$. Infection sites were pulmonary sites $(\mathrm{n}=28)$ and the paranasal sinuses $(\mathrm{n}=1)$. Filamentous fungi included Aspergillus spp. $(\mathrm{n}=24)$, Acremonium spp. $(\mathrm{n}=1)$, and Alternaria $\operatorname{spp}(\mathrm{n}=1)$. Active surveillance data after the outbreak (between May 1, 2011, and May 31, 2013) revealed 2 additional proven IFFI cases, 5 probable IFFI cases, and 4 possible IFFI cases (Figure 1).

\section{Matched Case-Control Investigation}

Among 284 potential control patients, only 102 could be matched to IFFI patients, for a mean of 3.5 controls per IFFI case. Of these, 49 control patients were matched to "proven" and "probable" IFFI patients. Control patients were hospitalized between November 1, 2008, and March 31, 2011. Table 1 shows the characteristics of IFFI patients and control patients. IFFI patients tended to have more acute and aggressive hematological diseases than control patients and to have received cytotoxic chemotherapy more often in the previous year. Corticosteroids were prescribed less often to IFFI patients than to control patients in the 3 previous months. IFFI patients were hospitalized for a longer period than control patients and had more radiation therapy during their hospital stays. IFFI patients developed severe neutropenia during hospital stay more frequently than control patients and for a longer duration. The mean number of transfers was significantly higher for IFFI patients, with more prescriptions for computed tomography scans, magnetic resonance imaging, pulmonary function assessment, and dermatology and/or stomatology visits. Depending on the location within the hospital, patients were transported through the lower ground floor corridors; IFFI patients were exposed more frequently than controls. The proportion of patients with transfers during an episode of neutropenia was significantly higher among IFFI patients than among control patients.

According to univariate analysis (Table 2, left), the odds of developing IFFI increased significantly by 8.6 -fold when the number of transfers was $>5$. According to multivariate analysis (Table 3, left), the adjusted odds of developing IFFI increased by 6.1 -fold when the number of transfers was $>5$. The odds of

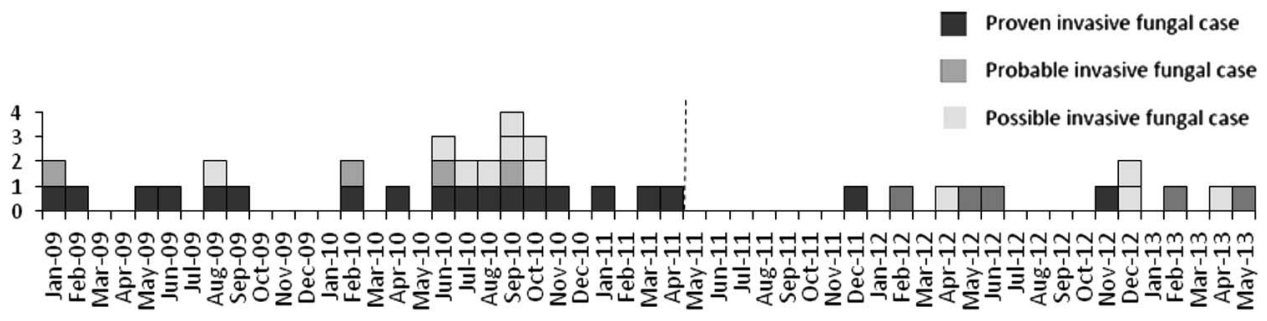

FIGURE 1. Epidemic curve of "proven," "probable," or “possible” invasive filamentous fungal infection between January 1, 2009, and April 31, 2011 and after the outbreak from May 1, 2011, to May 31, 2013, University of Geneva Hospitals, Geneva, Switzerland. The vertical dotted line illustrates the initiation of active surveillance of invasive filamentous fungal infection cases. 
TA BLE 1. Socio-demographic and Clinical Characteristics of Patients with Invasive Filamentous Fungal Infection Compared with Control Patients, University of Geneva Hospitals, Geneva, Switzerland, January 1, 2009, through April 31, 2011

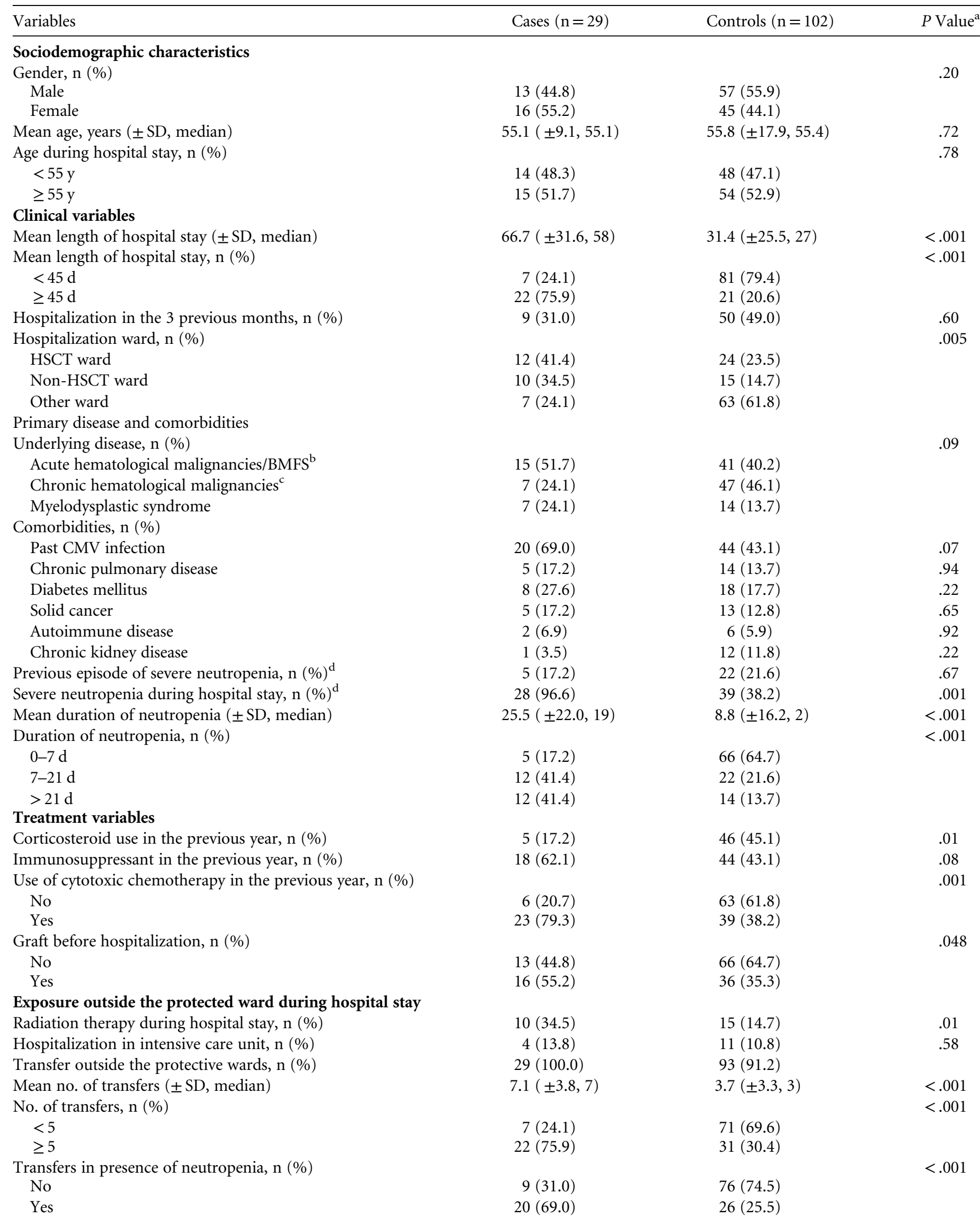


Table 1. Continued

\begin{tabular}{|c|c|c|c|}
\hline Variables & Cases $(n=29)$ & Controls $(n=102)$ & $P$ Value $^{\mathrm{a}}$ \\
\hline No. of transfers outside the main hospital building, $\mathrm{n}(\%)$ & $27(93.1)$ & $72(70.6)$ & .03 \\
\hline Returned home during hospital stay, n (\%) & $13(52.0)$ & $38(37.6)$ & .09 \\
\hline \multicolumn{4}{|l|}{ Outcomes } \\
\hline Vital status at the end of hospital stay, n (\%) & & & .09 \\
\hline Alive & $22(75.9)$ & $90(88.2)$ & \\
\hline Dead & $7(24.1)$ & $12(11.8)$ & \\
\hline
\end{tabular}

NOTE. SD, standard deviation; CMV, cytomegalovirus; HSCT, hematopoietic stem cell transplantation.

${ }^{\mathrm{a}} P$ values obtained from univariate conditional logistic regression models.

${ }^{\mathrm{b}}$ Acute myeloid leukemia, acute lymphoblastic leukemia, BMF, bone marrow failure syndrome, such as aplastic anemia.

${ }^{c}$ Chronic lymphatic leukemia, chronic myeloid leukemia, non-Hodgkin lymphoma, Hodgkin lymphoma, multiple myeloma, myeloproliferative neoplasm.

${ }^{\mathrm{d}}$ Severe neutropenia defined as an absolute neutrophil count $<200$ cells $/ \mu \mathrm{L}$.

developing IFFI was independently increased with a length of hospital stay $>45$ days and transfer during neutropenia and tended to be associated with an underlying hematological disease. In multivariate analysis, the odds of developing IFFI increased by 5.9 -fold when the number of transfers was $>5(P=.01)$, by 7.9-fold when the length of hospital stay was $>45$ days $(P=.001)$, and by 5.1 -fold when cytotoxic chemotherapy had been used during the previous year $(P=.03)$.

When excluding "possible" cases, the odds of IFFI was significantly increased by 16.7-fold when the number of transfers was $>5$ (Table 2, right) in univariate analysis, but this factor was not significant in multivariate analysis (Table 3, right). After adjusting for the length of hospital stay and the use of cytotoxic chemotherapy, the odds of developing an IFFI tended to increase by 7.5 -fold when the number of transfers was $>5$ without reaching significance $(P=.079)$.

\section{Environmental Surveillance}

Air cultures showed no significant difference in mean global growth across the 3 study periods $(P=.25)$ or in mean filamentous fungi growth $(P=.44)$. When stratified by ward (Tables $4 \mathrm{a}, \mathrm{b})$, a significant difference was observed in mean air growth cultures in the non-HSCT ward, leading to a global significant difference of mean air growth cultures between wards (Table 4a). There was no difference between wards regarding specific Aspergillus spp. growth (Table 4b). Notably, environmental surveillance is not implemented in the corridors used to transfer patients from the protective wards to elsewhere in the hospital.

\section{Outbreak Management and Control}

Due to the increased incidence of IFFI from January to July 2010, standard antifungal prophylaxis was changed from fluconazole $200 \mathrm{mg} /$ day to voriconazole $400 \mathrm{mg} /$ day for all patients admitted to the 2 wards from July 2010 until July 2011, when the standard antifungal prophylaxis was changed to posaconazole $200 \mathrm{mg} / 3$ times per day until April 2013.
Caspofungine $50 \mathrm{mg} /$ day was given in case of hepatic intolerance. All ventilation filters were changed. An airlock was installed in the entrance of the central corridor of the non-HSCT unit, as well as an automatic closure system for the doors of the standard rooms. Weekly environmental sampling (air and surface) was performed to document the possible presence of fungi in the protected wards. Additional cleaning measures were put in place for isolation rooms, including any computer equipment. Finally, preventive measures for at-risk patients (well-fitting face masks, gowns, caps, transportation, food, and objects made available to patients) were reinforced, and a protected bed for patient transport was provided. Additional costs of both preventive measures and treatment of IFFI were estimated at US $\$ 2,191,104$ between July 2010 and June 2011, representing an average of US\$27,372 per patient admitted; $74 \%$ of these costs were due to the change in antifungal prophylaxis.

\section{I S C USSION}

The occurrence of an outbreak of IFFI at our institution during summer 2010 could be explained by a combination of factors. The investigation confirmed our main hypothesis of an association between IFFI and exposure to construction work, represented by an increased number of medical in-hospital transfers during the hospital stay. IFFI was also independently associated with the presence of neutropenia during transfer and exposure to cytotoxic chemotherapy.

The relation between the occurrence of IFFI, especially invasive aspergillosis, and the presence of construction work has been well established. ${ }^{5,7,13,17-19}$ In brief, high concentrations of airborne fungal spores imply a consecutive colonization of pulmonary or sinus tissues and the subsequent development of invasive infection, particularly among immunocompromised hosts. ${ }^{20}$ Such an association has also been demonstrated in patients who were not severely immunocompromised but were possibly exposed to very large amounts of fungal spores or with transient immunodeficiency. ${ }^{9,21}$ The US Centers for Disease 
т А вLE 2. Risk Factors for Invasive Filamentous Fungal Infection (IFFI) by Univariate Conditional Logistic Regression on All Cases (left) and Restricted on "Proven" and "Probable" Cases (right) in the University of Geneva Hospitals, Geneva, Switzerland, January 1, 2009,

through April 31, 2011

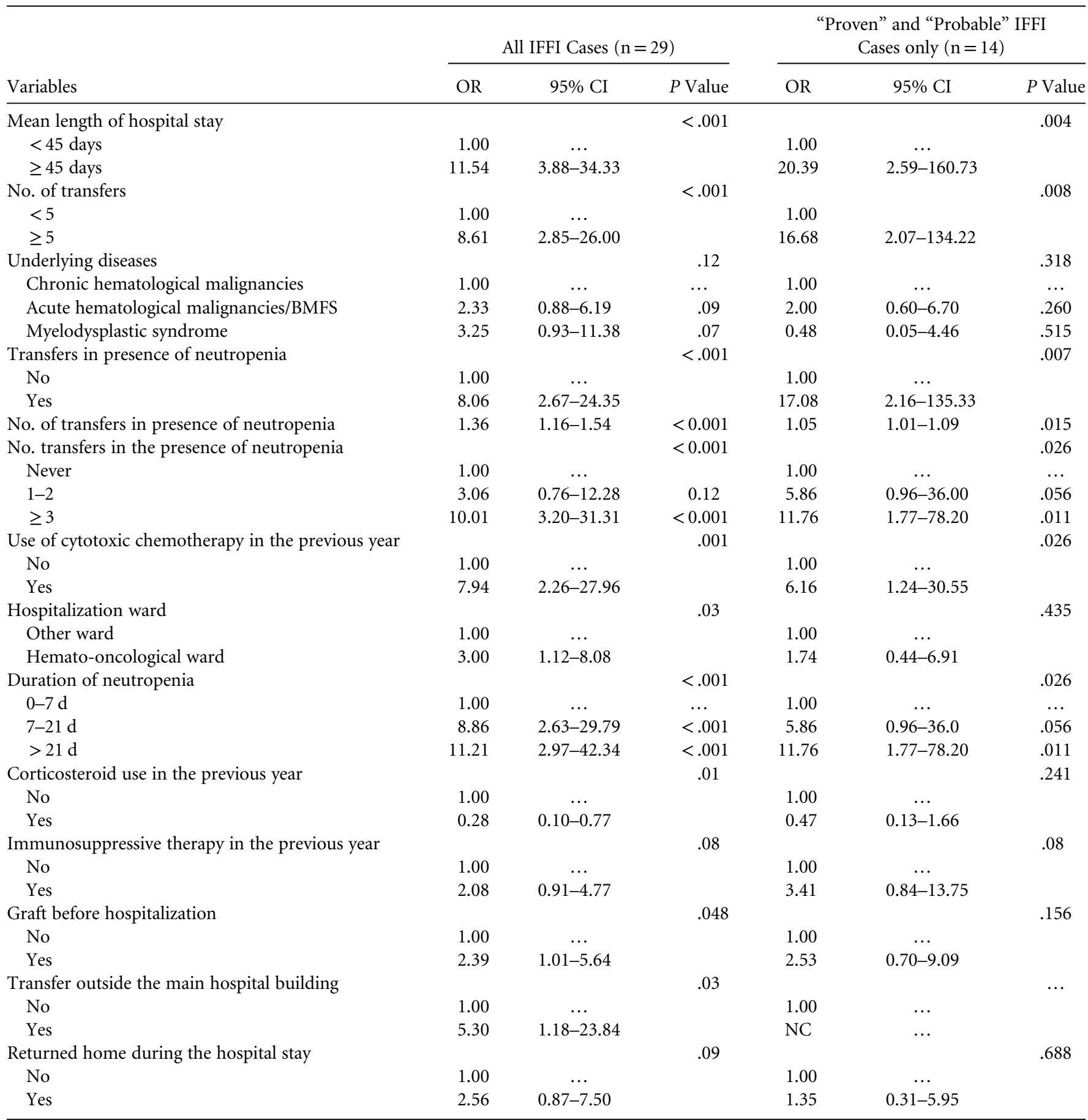

NOTE. CI, confidence interval; OR, odds ratio; BMFS, bone marrow failure syndrome; NC, statistical model did not converged due to null number in one of the subgroups.

Control and Prevention have made several recommendations: (1) avoid hospital construction and renovation areas whenever possible; (2) use high-efficiency particulate air filtration, directed room airflow, correctly sealed rooms, and a high rate of room air exchange ( $>12$ air changes/hour); (3) use barriers between patient care and construction areas; and (4) use high-efficiency masks on immunocompromised patients. ${ }^{22}$ Additional measures are recommended during building construction, ${ }^{6,12,14,23}$ 
TA A LE 3. Risk Factors for Invasive Filamentous Fungal Infection by Multivariate Conditional Logistic Regression on All Cases (left) and Restricted on "Proven" and "Probable" Cases (right) in the University of Geneva Hospitals, Geneva, Switzerland, January 1, 2009, through April 31, 2011

\begin{tabular}{|c|c|c|c|c|c|c|}
\hline \multirow[b]{2}{*}{ Variables } & \multicolumn{3}{|c|}{ All Cases $(n=29)^{a}$} & \multicolumn{3}{|c|}{$\begin{array}{l}\text { "Proven" and "Probable" } \\
\text { Cases Only }(\mathrm{n}=14)^{\mathrm{b}}\end{array}$} \\
\hline & OR & $95 \% \mathrm{CI}$ & $P$ Value & OR & $95 \% \mathrm{CI}$ & $P$ Value \\
\hline Mean length of hospital stay & & & .001 & & & .094 \\
\hline$<45 \mathrm{~d}$ & 1.00 & $\ldots$ & & 1.00 & $\ldots$ & \\
\hline$\geq 45 \mathrm{~d}$ & 10.22 & $2.51-41.56$ & & 23.54 & $0.58-947.66$ & \\
\hline$\geq 5$ & 6.11 & $1.25-29.92$ & & 2.29 & $0.19-27.19$ & \\
\hline Underlying diseases & & & .07 & & & .896 \\
\hline Chronic hematological malignancies & 1.00 & $\ldots$ & $\ldots$ & 1.00 & $\ldots$ & $\ldots$ \\
\hline Acute hematological malignancies/BMFS & 2.84 & $0.69-11.59$ & .15 & 1.90 & $0.10-37.19$ & .673 \\
\hline Myelodysplastic syndrome & 12.11 & $1.38-106.35$ & .02 & 2.69 & $0.03-224.36$ & .661 \\
\hline Transfers in presence of neutropenia & & & .04 & & & .062 \\
\hline
\end{tabular}

${ }^{\mathrm{a}}$ The pseudo-R2 of the multivariate model on all cases was $0.58(\mathrm{n}=131)$.

'The pseudo-R2 of the multivariate model excluding "possible" cases was $0.66(\mathrm{n}=.63)$.

table 4. Mean Total Growth (A) and Specific Aspergillus spp. Growth (B) on Air Cultures in the 2 Wards

A. Mean Growth on Air Cultures in $\mathrm{CFU} / \mathrm{m}^{3}$ per Ward during the 3 Study Periods

\begin{tabular}{|c|c|c|c|c|c|}
\hline & \multicolumn{2}{|l|}{ HSCT Ward } & \multicolumn{2}{|l|}{ Non-HSCT Ward } & \multirow[b]{2}{*}{ Global $P$ Value } \\
\hline & $\begin{array}{c}\text { Mean Growth in CFU } / \mathrm{m}^{3} \\
\text { (range, median) }\end{array}$ & $P$ Value $^{\mathrm{a}}$ & $\begin{array}{l}\text { Mean Growth in CFU } / \mathrm{m}^{3} \\
\text { (range, median) }\end{array}$ & $P$ Value $^{\mathrm{a}}$ & \\
\hline Before the epidemic & $19.43(0-220,5)$ & .63 & $9.43(0-145,5)$ & $<.001$ & $<.001$ \\
\hline During the epidemic & $18.98(0-205,0)$ & & $18.44(0-195,5)$ & & \\
\hline After the epidemic & $28.87(0-400,0)$ & & $57.85(0-450,20)$ & & \\
\hline
\end{tabular}

B. Mean Growth of Aspergillus spp. in Positive Samples on Air Cultures in CFU $/ \mathrm{m}^{3}$ per Ward during the 3 Study Periods

\begin{tabular}{|c|c|c|c|c|c|}
\hline & \multicolumn{2}{|l|}{ HSCT Ward } & \multicolumn{2}{|l|}{ Non-HSCT Ward } & \multirow[b]{2}{*}{ Global $P$ Value } \\
\hline & $\begin{array}{c}\text { Mean growth in } \mathrm{CFU} / \mathrm{m}^{3} \\
\text { (range, median) }\end{array}$ & $P$ Value $^{\mathrm{c}}$ & $\begin{array}{l}\text { Mean growth in } \mathrm{CFU} / \mathrm{m}^{3} \\
\text { (range, median) }\end{array}$ & $P$ Value $^{\mathrm{c}}$ & \\
\hline Before epidemic & $2.27(0-45,0)$ & .65 & $2.08(0-10,0)$ & .55 & .77 \\
\hline During epidemic & $0.45(0-5,0)$ & & $0.83(0-5,0)$ & & \\
\hline After epidemic & $0.29(0-10,0)$ & & $0.46(0-10,0)$ & & \\
\hline
\end{tabular}

NOTE. CFU, colony-forming units; HSCT, hematopoietic stem cell transplantation.

${ }^{a}$ Due to nonnormal distribution, the Kruskal-Wallis test was applied.

${ }^{\mathrm{b}} P$ value comparing the mean growth on air cultures between HSCT and non-HSCT wards adjusted for study periods using Poisson regression.

${ }^{c}$ Due to nonnormal distribution, Kruskal-Wallis test was applied.

${ }^{\mathrm{d}} P$ value comparing the count of positive growth on air cultures between HSCT and non-HSCT wards adjusted on study periods using Poisson regression.

including misting of the construction area with water to minimize dust generation and protection of walkways from demolition and/or construction sites. All of these recommendations were strictly applied at our institution from the end of
2008, when several wards where exposed to construction work. Protective measures were strengthened, and medical care teams were instructed to recommend mask wear to immunocompromised patients as soon as they arrived in the vicinity of the 
hospital until they left the hospital. Windows of wards located near construction sites always remained closed. Notably, the outbreak occurred during the demolition in February 2010 of an adjoining building and the construction of a new one on the same site. In addition, a new large construction was built during this time on a site near our facility. However, although mean total growth on air and surface cultures were higher in the 2 wards after the occurrence of the outbreak, there was a lower number of IFFI cases during this period, suggesting that the preventive measures applied were effective. The only suspected defect in preventive measures was the organization of patient transfer outside the patient's room. During specific transfers to radiology or elsewhere, patients were transported through the lower ground floor corridors of the hospital. Although all preventive measures were strictly applied in oncohematology, the use of high-efficiency masks during transport had not been strictly audited, and we suspect that this might explain the increase of IFFI in patients exposed to airborne fungal spores related to proximal construction areas. ${ }^{24,25}$ To test our hypothesis, we quantified the total number of transfers for both IFFI patients and control patients during their hospital stay and during neutropenia; this analysis revealed a positive association between IFFI and both variables. We were unable to retrieve any information in medical records related to the use of specific protective masks during patient transport. However, following the outbreak, 2 medical students blindly observed 6 immunocompromised patients hospitalized in 1 of the 2 wards who all failed the fit test. ${ }^{26}$ The independent associations of a high number of transfers and the presence of neutropenia suggest that IFFI patients were probably not sufficiently protected during transport in the corridors. The combination of exposure to fungal spores enhances the risk of IFFI. ${ }^{27,28}$

We are aware of several limitations of our study. First, similar to other retrospective studies, we could not exclude the existence of nondifferential information bias due to missing data both in IFFI patients and control patients regarding the use of a mask during transport outside the protected wards. To counterbalance this lack of information and to test the hypothesis for an association between IFFI and exposure to air contamination by construction work, we used a surrogate objective measure quantifying the number of transfers. Although we had data on environmental surveillance in the 2 protected wards, we had no data regarding potential environmental contamination in the suspected corridors. Second, antifungal prophylaxis was broadened to include filamentous fungi from July 2010, but due to the matching of control patients with IFFI patients on the period of hospitalization, it did not bias the estimation of the association between IFFI and the risk factors tested. Third, we categorized chemotherapy into "highly aggressive," "moderately aggressive," and "less aggressive" and dichotomized the variable into cytotoxic or noncytotoxic chemotherapy. To minimize observer expectation bias, an independent physician (unaware of case-control status) established this classification from the list of drugs prescribed to patients. Finally, we did not observe any deaths among possible cases, suggesting early diagnosis with improved outcome or overdiagnosis of IFFI in the outbreak context. The association between IFFI and transfer outside the protected ward was preserved when excluding possible cases but did not reach significance in the multivariate models due to a lack of power.

Our results demonstrate that even if recommendations for environmental control are applied in a well-organized teaching hospital, process control should consider all steps of patient care management, from hospitalization in a specific ward to all interactions between the patient, various consultation services, and the hospital environment. All healthcare workers interacting with immunocompromised patients should receive specific education regarding the preventive measures (especially the use of high-efficiency masks and control of their fit) and behaviors to adopt in these specific situations. All of these measures were reinforced after the investigation of the outbreak, and this led to a significant decrease in the number of IFFI cases after May 2011.

In conclusion, IFFI represents an opportunistic complication of concern among immunocompromised patients with hematological malignancies with a $21 \%(8 \%-40 \%)$ associated mortality in our study and important extra costs. Intrinsic individual risk factors seem difficult to control, but more importance should be given to protective measures, especially the use of a high-efficiency mask during transport outside the protected environment of the dedicated ward and the avoidance of unnecessary transfers.

\section{ACKNOWLEDGMENTS}

We thank Rosemary Sudan for editorial assistance, Véronique Camus for help in the management of the outbreak, Dr. Walter Zingg for help in the use of environmental surveillance data, Gilles Cohen for the retrieval of the patient list, Cyril Pecora for providing the plan of the wards, and David Pecoul and Mihaela Munteanu for observations regarding patients.

Financial support: This work was supported by an institutional grant of the University of Geneva Hospitals (\#2011.03).

Potential conflicts of interest: All authors report no conflicts of interest. All authors have submitted the ICMJE Form for Disclosure of Potential Conflicts of Interest. Conflicts that the editors consider relevant to the content of the manuscript have been disclosed.

Address correspondence to Professor Didier Pittet, MD, MS, Infection Control Program and WHO Collaborating Centre on Patient Safety, University of Geneva Hospitals, 4 Rue Gabrielle-Perret-Gentil 1211 Geneva 14, Switzerland (didier.pittet@hcuge.ch).

\section{REFERENCES}

1. Lin SJ, Schranz J, Teutsch SM. Aspergillosis case-fatality rate: systematic review of the literature. Clin Infect Dis 2001;32:358-366.

2. Gavalda J, Len O, San Juan R, et al. Risk factors for invasive aspergillosis in solid-organ transplant recipients: a casecontrol study. Clin Infect Dis 2005;41:52-59.

3. Michallet M, Ito JI. Approaches to the management of invasive fungal infections in hematologic malignancy and hematopoietic cell transplantation. J Clin Oncol 2009;27:3398-3409. 
4. Marr KA, Carter RA, Crippa F, Wald A, Corey L. Epidemiology and outcome of mould infections in hematopoietic stem cell transplant recipients. Clin Infect Dis 2002;34:909-917.

5. McCann S, Byrne JL, Rovira M, et al. Outbreaks of infectious diseases in stem cell transplant units: a silent cause of death for patients and transplant programmes. Bone Marrow Transplant 2004;33:519-529.

6. Chang CC, Athan E, Morrissey CO, Slavin MA. Preventing invasive fungal infection during hospital building works. Intern Med J 2008;38:538-541.

7. Vonberg RP, Gastmeier P. Nosocomial aspergillosis in outbreak settings. J Hosp Infect 2006;63:246-254.

8. Kaiser L, Huguenin T, Lew PD, Chapuis B, Pittet D. Invasive aspergillosis. Clinical features of 35 proven cases at a single institution. Medicine (Baltimore) 1998;77:188-194.

9. Pittet D, Huguenin T, Dharan S, et al. Unusual cause of lethal pulmonary aspergillosis in patients with chronic obstructive pulmonary disease. Am J Respir Crit Care Med 1996;154:541-544.

10. Eggimann P, Chevrolet JC, Starobinski M, et al. Primary invasive aspergillosis of the digestive tract: report of two cases and review of the literature. Infection 2006;34:333-388.

11. De Pauw B, Walsh TJ, Donnelly JP, et al. Revised definitions of invasive fungal disease from the European Organization for Research and Treatment of Cancer/Invasive Fungal Infections Cooperative Group and the National Institute of Allergy and Infectious Diseases Mycoses Study Group (EORTC/MSG) Consensus Group. Clin Infect Dis 2008;46:1813-1821.

12. Berthelot P, Loulergue P, Raberin H, et al. Efficacy of environmental measures to decrease the risk of hospital-acquired aspergillosis in patients hospitalised in haematology wards. Clin Microbiol Infect 2006;12:738-744.

13. Chang CC, Cheng AC, Devitt B, et al. Successful control of an outbreak of invasive aspergillosis in a regional haematology unit during hospital construction works. J Hosp Infect 2008;69:33-38.

14. Sehulster L, Chinn RY. Guidelines for environmental infection control in health-care facilities. Recommendations of CDC and the Healthcare Infection Control Practices Advisory Committee (HICPAC). MMWR Recomm Rep 2003;52:1-42.

15. Hennessy S, Bilker WB, Berlin JA, Strom BL. Factors influencing the optimal control-to-case ratio in matched case-control studies. Am J Epidemiol 1999;149:195-197.

16. Courvoisier DS, Combescure C, Agoritsas T, Gayet-Ageron A, Perneger TV. Performance of logistic regression modeling: beyond the number of events per variable, the role of data structure. J Clin Epidemiol 2011;64:993-1000.
17. Mahieu LM, De Dooy JJ, Van Laer FA, Jansens H, Ieven MM. A prospective study on factors influencing aspergillus spore load in the air during renovation works in a neonatal intensive care unit. J Hosp Infect 2000;45:191-197.

18. Raviv Y, Kramer MR, Amital A, Rubinovitch B, Bishara J, Shitrit D. Outbreak of aspergillosis infections among lung transplant recipients. Transpl Int 2007;20:135-140.

19. Anderson K, Morris G, Kennedy H, et al. Aspergillosis in immunocompromised paediatric patients: associations with building hygiene, design, and indoor air. Thorax 1996;51:256-261.

20. Benet T, Nicolle MC, Thiebaut A, et al. Reduction of invasive aspergillosis incidence among immunocompromised patients after control of environmental exposure. Clin Infect Dis 2007;45:682-686.

21. Pelaez T, Munoz P, Guinea J, et al. Outbreak of invasive aspergillosis after major heart surgery caused by spores in the air of the intensive care unit. Clin Infect Dis 2012;54:e24-e31.

22. Guidelines for preventing opportunistic infections among hematopoietic stem cell transplant recipients. MMWR Recomm Rep 2000;49:1-125.

23. VandenBergh MF, Verweij PE, Voss A. Epidemiology of nosocomial fungal infections: invasive aspergillosis and the environment. Diagn Microbiol Infect Dis 1999;34:221-227.

24. Bouza E, Pelaez T, Perez-Molina J, et al. Demolition of a hospital building by controlled explosion: the impact on filamentous fungal load in internal and external air. J Hosp Infect 2002;52: 234-242.

25. Iwen PC, Davis JC, Reed EC, Winfield BA, Hinrichs SH. Airborne fungal spore monitoring in a protective environment during hospital construction, and correlation with an outbreak of invasive aspergillosis. Infect Control Hosp Epidemiol 1994;15:303-306.

26. Pecoul D, Munteanu M, Iten A, Pittet D. Which preventive measures are taken for immunocompromised patients during their transport outside the ward? In: Program and abstracts of the 1stInternational Conference on Prevention and Infection Control (ICPIC) June 29-July 2 2011; Geneva, Switzerland. Abstract 383.

27. Weber SF, Peacock JE Jr., Do KA, Cruz JM, Powell BL, Capizzi RL. Interaction of granulocytopenia and construction activity as risk factors for nosocomial invasive filamentous fungal disease in patients with hematologic disorders. Infect Control Hosp Epidemiol 1990;11:235-242.

28. Panackal AA, Dahlman A, Keil KT, et al. Outbreak of invasive aspergillosis among renal transplant recipients. Transplantation 2003;75:1050-1053. 